\title{
Overlapping neural response to the pain or harm of people, animals, and nature
}

Vani A. Mathur ${ }^{1,2}$, Bobby K. Cheon ${ }^{1,3}$, Tokiko Harada ${ }^{1,4}$, Jason M. Scimeca ${ }^{1,5}$, Joan Y. Chiao ${ }^{1}$

${ }^{1}$ Department of Psychology, Northwestern University, 2029 Sheridan Road, Evanston, IL 60208, USA

${ }^{2}$ Present affiliation: Department of Psychology, Texas A\&M University, College Station, TX 77843-4235, USA

${ }^{3}$ Present affiliation: Division of Psychology Nanyang Technological University and Clinical Nutrition Research Center, Singapore Institute for Clinical Sciences (A*Star), Singapore

${ }^{4}$ Present affiliation: National Institute for Physiological Sciences, 38 Nishigonaka Myodaiji, Okazaki, Japan 444-8585

${ }^{5}$ Present affiliation: Department of Cognitive, Linguistic, and Psychological Sciences, Brown University, 190 Thayer Street, Providence, RI 02912, USA

Sources of support: This research was supported by National Science Foundation grants BCS0720312 and BCS-0722326 to J.Y.C., and manuscript preparation was supported by the National Institutes of Health Ruth L. Kirschstein National Research Service Award T32 NS070201 to V.A.M. 


\begin{abstract}
Interpersonal pain perception is a fundamental and evolutionarily beneficial social process. While critical for navigating the social world, whether or not people rely on similar processes to perceive and respond to the harm of the non-human biological world remains largely unknown. Here we investigate whether neural reactivity toward the suffering of other people is distinct from or overlapping with the neural response to pain and harm inflicted upon non-human entities, specifically animals and nature. We used fMRI to measure neural activity while participants $(n=15)$ perceived and reported how badly they felt for the pain or harm of humans, animals, and nature, relative to neutral situations. Neural regions associated with perceiving the pain of other people (e.g. dorsal anterior cingulate cortex, bilateral anterior insula) were similarly recruited when perceiving and responding to painful scenes across people, animals, and nature. These results suggest that similar brain responses are relied upon when perceiving the harm of social and non-social biological entities, broadly construed, and that activity within the dorsal anterior cingulate cortex and bilateral anterior insula in response to pain-relevant stimuli is not uniquely specific to human targets.
\end{abstract}

Keywords: Pain perception, empathy, fMRI, anterior cingulate cortex, insula Abstract word count: 179

Main text word count: 5695

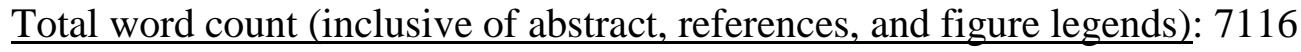


NEURAL RESPONSE TO PAIN OF PEOPLE, ANIMALS AND NATURE

\section{Introduction}

Around the world, people donate hundreds of billions of dollars each year to regional and international charities ranging from the American Red Cross and Doctors without Borders to the Nature Conservancy and World Wildlife Fund (Charities Aid Foundation, 2011). While a majority of donations fund efforts to directly relieve human suffering, people also routinely fund efforts to relieve non-human harm and suffering, such as animal rescue and nature conservation. Evolutionary psychologists have long argued that altruistic acts such as charitable donation emerge from the fundamental capacity for people to perceive and feel the suffering of others and act to alleviate that suffering (i.e., perception-action model of empathy, Preston $\&$ de Waal, 2002).

A core network of brain regions underlies the recognition and interpretation of the pain of others (e.g. meta-analyses by Fan, Duncan, Greck \& Northoff, 2011 and Lamm, Decety, \& Singer, 2011). Specifically, the dorsal anterior cingulate (dACC) and bilateral anterior insular (AI) cortices are recruited when perceiving the pain of others across diverse contexts, such as physical pain or injury (Jackson, Rainville, \& Decety, 2006; Lamm, Nusbaum, Meltzoff, \& Decety, 2007; Singer et al., 2004; Zaki, Ochsner, Hanelin, Wager, \& Mackey, 2007), painful facial expressions (Botvinick et al., 2005; Saarela, Hlushchuk, Williams, Schurmann, Kalso, \& Hari, 2007), social pain of exclusion (Masten, Morelli, \& Eisenberger, 2011), and complex emotional pain (Cheon et al., 2011; Chiao, Mathur, Harada, Lipke, 2009; Mathur, Harada, Lipke, \& Chiao, 2010). Although numerous prior studies have demonstrated and described the neural basis of the perception of human suffering, the specificity of the responding of this system is not well understood. Given the prevalence of costly helping behaviors directed to relieve the pain or 
NEURAL RESPONSE TO PAIN OF PEOPLE, ANIMALS AND NATURE

harm of animals and nature, the neural processes recruited to perceive and respond to such nonhuman targets are of particular interest.

Environmental preservation campaigns often rely upon a bidirectional relationship between empathy and anthropomorphism, the human tendency to attribute agency or human characteristics (e.g., emotions and motivation) to non-human targets (Epley, Waytz, Cacioppo, 2007; Herrmann, Waxman, Medin, 2010). One such campaign asked "If your mother were Texas, would you still litter?” (Texas Department of Transportation, 2001). Similarly, animal protection agencies often show hurt animals in advertisements to motivate helping behavior and financial giving (e.g. The Humane Society of the United States, 2011). Prior behavioral research suggests that people perceive pain among, and feel empathy for, physically and emotionally hurt animals (Phillips, 2009; Schultz, 2000). Given the human propensity to empathize with nonhuman entities, such as animals and nature, one hypothesis is that perception of pain and suffering may rely on a shared neural network across both human and non-human entities. Supporting this hypothesis, the one study to examine neural response to the pain of a non-human target (i.e., dogs) found overlapping neural response within the dACC and bilateral AI while passively viewing the pain of dogs and humans (Franklin et al., 2013).

On the other hand, prior evidence suggests that neural processing of the pain of other people may be distinct from processing the pain or harm of animals or the natural environment. For instance, people recruit different brain areas when visually processing and making inferences about other people relative to when making similar inferences about non-human events (Mitchell, Macrae, \& Banaji, 2005; Saxe \& Kanwisher, 2003). Individual differences in trait empathy have also been associated with activity in social cognitive brain regions when viewing human, but not non-human, natural scenes (Wagner, Kelley, \& Heatherton, 2011). Therefore, 
NEURAL RESPONSE TO PAIN OF PEOPLE, ANIMALS AND NATURE

human perception of the pain of other people may rely upon a distinct network of brain regions compared to the perception of pain or harm of animals or the environment.

A third possibility is that neural processing of the pain or harm of human and non-human entities relies on the same neural regions, but to differing extents within these regions.

Supporting this view, prior behavioral and psychophysiological evidence shows that people display greater empathic and physiological reactivity for pain of animals that are phylogenetically closer to humans (Rae Westbury \& Neumann, 2008). Furthermore, strength of belief that animals have mental capacity (and therefore may be more similar to humans) correlates with self-reported empathy for animals (Hills, 1995). Schultz (2001) suggested that empathic concern for the environment varies across individuals to the extent that they include and integrate the natural environment as part of the self. When people were instructed to take the perspective of animals being harmed by pollution in the environment, they subsequently reported greater empathy for the natural environment relative to people who objectively viewed the same scenes (Schultz, 2000). Consequently, people may exhibit differential psychological and neural responses when encountering pain or harm inflicted upon human relative to various non-human targets (i.e. animals or the environment) to the extent that they perceive the targets to be similar to themselves. Perceived similarity and degree of identification with a human target has also been shown to modulate neural responses underlying pain perception and empathy (Decety, Echols, \& Correll, 2010; Mathur, Harada, \& Chiao, 2012). One study found that when viewing and perceiving the pain of racial ingroup, relative to outgroup, targets in pain, Chinese and Caucasian participants show increased neural activity in the dACC and left frontal cortex (Xu, Zuo, Wang, \& Han, 2009). However, other studies have found no difference in dACC and AI activity in response to the emotional pain of racial ingroup, relative to outgroup, people among 
Caucasian and African Americans (Mathur et al., 2010) and native Koreans and Caucasian Americans (Cheon, et al., 2011). Taken together, the above evidence suggests that neural processing associated with pain perception may be expected to differ between human and nonhuman entities due to variations in characteristics such as humanness, perceived agency, phylogenetic similarity, as well as the degree to which perceivers identify with the target. This alternative hypothesis would be supported by results demonstrating different levels of reactivity in pain processing regions, in response to the pain or harm of humans, animals, and nature.

In addition to our main hypotheses, this study was also motivated by an interest in the flexibility and specificity of dACC and AI reactivity in the context of perceiving pain or harm ${ }^{1}$. Determining whether AACC and AI reactivity extends to the perception of harm inflicted on nonhuman targets that may not have subjective experiences (i.e. nature) may elucidate whether $\mathrm{dACC}$ and AI activity during pain perception reflects empathic resonance with the subjective experience of the pain of another, or suggest this activity may instead subserve a broader psychological process in response pain or harm perception itself. If dACC and AI reactivity is exclusively observed in response to the suffering of human targets, but not for non-human targets, then activity within $\mathrm{AACC}$ and AI may be reflecting processes that are specific to interpersonal and social contexts rather than a reflection of pain-relevant processing. Yet, if dACC and AI reactivity is broadly observed in response to the suffering of all targets, then this activity may subserve broader processes of pain or harm perception itself. Given the broad functions associated with the AI and AACC across social, physiological, and emotional processes, this information would be critical for specifying the AI and dACC's potential role in empathic and pain-relevant processing. 
NEURAL RESPONSE TO PAIN OF PEOPLE, ANIMALS AND NATURE

To test these hypotheses, we investigated whether distinct or shared neural circuitry is recruited when empathizing with people, animals, and nature in either painful or neutral scenarios, and whether degree of neural reactivity varies as a function of target group. Here, we use the term empathy to refer to the subjective affective response and underlying neural response assessed while participants viewed pictures of the pain or harm of people, animals, or nature, and rated "How badly [they felt]" for each target.

\section{Methods}

\subsection{Participants}

Fifteen right-handed Caucasian volunteers ( 8 female, $M=25.3$ years, $S E=4.7$ ), with normal or corrected-to-normal vision participated in this study, and were compensated $\$ 25$ per hour of their time. All participants were healthy: free from chronic illness (including neurological, psychiatric, or substance-abuse conditions) and medications that alter blood flow or brain function. This study was approved by the Northwestern University Institutional Review Board, and informed written consent was obtained from each participant prior to the experiment.

\subsection{Stimuli}

Stimuli consisted of naturalistic visual scenes (640 pixels x 480 pixels) depicting people (Caucasian Americans), animals (various farm animals), or nature (plant life and natural waterways) under egregiously negative (e.g. people in the midst of a natural disaster such as a hurricane, injured or sick animals, deforestation or pollution) or neutral conditions (Figure 1). Twenty-four unique scenes were included for each of the six conditions (People Pain ${ }^{2}$, People Neutral, Animals Pain, Animals Neutral, Nature Pain, and Nature Neutral).

\section{$\underline{\text { 2.2.1 Stimuli validation }}$}


NEURAL RESPONSE TO PAIN OF PEOPLE, ANIMALS AND NATURE

In a separate stimuli validation study (results presented in Table 1), 27 participants (20 female, $M=23.4$ years, $S E=0.72)$ rated degree of perceived pain (How much pain do you think [this person, this animal, nature] is in?), level of perceived agency (How much control do you think [this person, this animal, nature] has over this situation?), and empathy felt (How badly do you feel for [this person, this animal, nature]?) for the person, animal, or nature in each scenario using an 8-point Likert scale $(0=$ not at all to $7=$ very much $)$.

\subsubsection{Perceived pain}

Results from a 2 (valence of experience: Pain, Neutral) x 3 (target group: People,

Animals, Nature) analysis of variance revealed a main effect of target group (People, Animals, or Nature), $F(2,52)=3.38, p=0.04$, and a main effect of valence, $F(1,26)=679.23, p<0.001$, on perceived pain ratings. People $[t(26)=25.66, p<0.001, d=5.65]$, animals $[t(26)=18.00, p<$ $0.001, d=4.26]$, and nature $[t(26)=17.35, p<0.001, d=4.11]$ were perceived as in significantly more pain in the Pain relative to the Neutral scenes. There was no significant interaction between target group and valence.

\subsubsection{Perceived agency}

Results from a 2 (valence of experience: Pain, Neutral) x 3 (target group: People, Animals, Nature) analysis of variance on perceived target agency ratings revealed main effects of target group $F(2,52)=66.24, p<0.001$, and valence $F(1,26)=8.30, p=0.008$, and a significant interaction between group and valence, $F(2,52)=29.77, p<0.001$. Both people and animals in pain were seen as having decreased agency (less power or control over their situation) than people and animals in neutral conditions [People: $t(26)=-4.94, p<0.001, d=-1.08$; $\underline{\text { Animals: } t}$ $(26)=-2.23, p=0.04, d=-0.57]$. There was no significant difference in agency ratings between Pain and Neutral nature scenes $(p>0.05)$. 


\subsubsection{Evoked empathy}

Correspondingly, there were main effects of target group (People, Animals, or Nature), $F(2,52)=32.94, p<0.001$, and valence, $F(1,26)=882.19, p<0.001$, on empathy ratings and a significant interaction between target group and valence $F(2,52)=6.37, p=0.003$, such that participants reported increased relative empathy (Pain - Neutral) toward animals compared to people $[t(26)=2.18, p=0.04, d=0.59]$ and nature $[t(26)=2.92, p=0.007, d=0.81]$, and participants reported increased empathy across domains toward pain relative to neutral conditions [ $\underline{\text { People: }} t(26)=22.73, p<0.001, d=4.99$; $\underline{\text { Animals: }} t(26)=33.19, p<0.001, d=$ 5.67; Nature: $t(26)=14.58, p<0.001, d=3.54]$, (Table 1). There was no significant difference in relative empathy ratings toward people and nature $(p>0.05)$.

\subsubsection{Validation study conclusion}

People and animals in Pain were seen as having less control over their situation (agency), than corresponding Neutral targets. As intended, targets in Pain stimuli were perceived to be in more pain than targets in Neutral stimuli, across target types. Accordingly, participants reported feeling worse (greater empathy) for targets in the Pain, relative to Neutral, stimuli, across target types (Table 1). We concluded that all Pain stimuli were seen as more painful than Neutral stimuli, and effectively evoked an affective response among participants.

Despite reporting perceiving pain and feeling badly for nature in Pain scenes, participants did not find nature in Pain more agentic than nature in Neutral scenes, suggesting that participants were not personifying nature. Though we did not asses directly personification or anthropomorphism, this result suggests that participants' empathy ratings are not necessarily dependent upon humanizing the non-human targets.

\subsection{Design}


NEURAL RESPONSE TO PAIN OF PEOPLE, ANIMALS AND NATURE

We employed a block design consisting of six types of task blocks depicting people, animals, or nature in pain or neutral scenarios. There were four blocks per task type. Each block consisted of six unique trials of that block type. Two presentation sequences were created and participants were randomly assigned to a sequence order at the time of scheduling. Within each of these sequences, task blocks were displayed in a fixed random order. There were no differences in any outcome variables based on sequence order. Additionally, task blocks were separated by control blocks during which participants pressed a button with their right index finger when a simple gray square appeared. These control blocks were not included as a contrast of interest in the subsequent analyses. For each trial, a complex visual scene or a gray square was displayed for $2500 \mathrm{~ms}$, followed by a black screen for 500ms.

For the current study we modified a block design which successfully induced empathic neural responses to the emotional suffering of other people (Mathur et al., 2010). We chose a block-design as they are generally more sensitive to the detection of relatively activated areas (e.g. areas more active in response to people vs. animals) and more statistically powerful than event-related designs (Amaro \& Barker, 2006; Birn, Cox, \& Bandettini, 2002).

\subsection{Procedure}

During scanning, participants responded to the question "How badly do you feel for the [person, animal, environment] in this scene?" on a four-point Likert scale $(1=$ not at all to $4=$ very much) using the corresponding number of button presses. This question has been used in prior studies examining empathy and neural empathic processes in response to the pain of other people (e.g. Mathur et al., 2010). This question was chosen to assess an affective response to the pain or harm of the targets. We refer to this process as empathy in the current paper, but note that participants were not instructed to perspective take, anthropomorphize, or ascribe feeling states 
NEURAL RESPONSE TO PAIN OF PEOPLE, ANIMALS AND NATURE

to the targets, but rather report their own subjective response toward each target. Participants were instructed to give their gut response rapidly upon seeing each scene. Prior to entering the scanner, participants were shown example scenes and given a practice trial in order to become familiar with the task.

Outside of the scanner, all participants were asked to complete the Interpersonal Reactivity Index (IRI), as a measure of trait empathy (Davis, 1980).

\subsection{Behavioral statistical analysis}

To determine if pain and target group affected on online empathy ratings and response reaction time, 2 (valence of experience: Pain, Neutral) x 3 (target group: People, Animals, Nature) repeated measures analyses of variance (ANOVA) were conducted.

To determine the relationship between empathy felt for people, animals, and nature, Pearson correlation analysis was conducted on these ratings.

\subsection{Imaging parameters}

Functional whole-brain images were acquired using a 3T Siemens Trio at the Center for Advanced Magnetic Resonance Imaging (CAMRI) facility located at the Northwestern University Medical School in Chicago, IL, USA. We acquired functional images by using T2*_ weighted, gradient echo, echo planar imaging sequences [repetition time $(\mathrm{TR})=2000 \mathrm{~ms}$; echo time $(\mathrm{TE})=25 \mathrm{~ms}$; flip angle $=70^{\circ} ; \mathrm{FOV}=20 \mathrm{~cm}, 64 \times 64$ matrix; 34 slices; $4 \mathrm{~mm}$ slice thickness (no gap); in-plane resolution $=3.0 \times 3.0 \mathrm{~mm}]$. A high-resolution anatomical T1-weighted image was also acquired $\left[\mathrm{TR}=2300 \mathrm{~ms} ; \mathrm{TE}=2.91 \mathrm{~ms} ;\right.$ flip angle $=9^{\circ} ; \mathrm{FOV}=256 \mathrm{~mm} ; 256 \times 256$ matrix; 160 slices; voxel size $=1.0 \times 1.0 \times 1.0 \mathrm{~mm}$ ] for each participant. All stimuli were presented using Presentation software (Neurobehavioral Systems, Albany, CA) and projected 
NEURAL RESPONSE TO PAIN OF PEOPLE, ANIMALS AND NATURE

onto a half-transparent viewing screen located behind the head coil. Participants viewed the stimuli using a tilted mirror attached to the head coil.

\subsection{Imaging processing and statistical analysis}

Functional images were analyzed using SPM2 software (Wellcome Department of Imaging Neuroscience, London, UK) implemented in Matlab (Mathworks, Cherborn, MA, USA). The first 6 volumes were discarded due to unsteady magnetization, and all of the remaining volumes were realigned spatially to the first volume and a mean image was created. After a high-resolution image was coregistered onto the mean image, all volumes were normalized to the MNI (Montreal Neurological Institute) space using a transformation matrix obtained from the normalization process of the high-resolution image of each individual participant to the MNI template. The normalized images were then spatially smoothed with an 8mm Gaussian kernel.

After preprocessing, statistical analysis for each individual participant was conducted using the general linear model (Friston et al., 1994). At the first level, each block of trials was modeled by convolving with a hemodynamic response function. For each participant, a linear regressor was applied to filter noise. In the subtraction analysis, 6 task conditions [People Pain, People Neutral, Animals Pain, Animals Neutral, Nature Pain, and Nature Neutral] were modeled separately, including fixation. Random effects analyses were conducted by averaging the contrast images for each effect of interest.

To identify the main effect of pain, whole-brain voxel-wise analyses were performed across target groups (People, Animals, Nature) on the simple contrast [Pain > Neutral] with a threshold of $p<0.05$ (FWE corrected), extant threshold = 10 voxels (Table 3 ), and within target 
NEURAL RESPONSE TO PAIN OF PEOPLE, ANIMALS AND NATURE

groups with a threshold of $p<0.005$ (FDR corrected), extant threshold $=10$ voxels (Tables 4-6, Figure 1).

To identify the main effect of target group on neural activity, and identify differences in neural response to people, animals, and nature, independent of valence of experience, a wholebrain voxel-wise analysis was performed on the simple contrasts [People (Pain + Neutral) $>$ Animals $($ Pain + Neutral $)],[$ People $($ Pain + Neutral $)>$ Nature $($ Pain + Neutral $)],[$ Animals $($ Pain + Neutral $)>$ Nature $($ Pain + Neutral) $]\left(\right.$ Table S1), [Animals ${ }_{(\text {Pain }+ \text { Neutral })}>$ People $\left._{(\text {Pain }+ \text { Neutral })}\right],\left[\right.$ Nature ${ }_{(\text {Pain }+ \text { Neutral })}>$ Animals (Pain + Neutral) $],[$ Nature $($ Pain + Neutral $)>$ People $($ Pain + Neutral) $]$, with a threshold of $p<0.005$ (FDR corrected), extant threshold $=10$ voxels.

Whole-brain voxel-wise analyses were also performed to identify possible pain by target group interactions in neural activity that would suggest differential neural response to pain or harm, relative to neutral situations, depending on target type [People (Pain - Neutral) $>$ Animals (Pain Neutral) $],\left[\right.$ People (Pain - Neutral) $>$ Nature $_{(\text {Pain - Neutral) }),[\text { Animals }}^{(\text {Pain - Neutral) }}>$ Nature $($ Pain - Neutral) $]$, [Animals $_{(\text {Pain - Neutral) }}>$ People $\left._{(\text {Pain - Neutral) }}\right]_{,}$[Nature $_{(\text {Pain - Neutral) }}>$ Animals (Pain - Neutral) $_{\text {, }}$ [Nature (Pain - Neutral) $>$ People (Pain - Neutral)], with a threshold of $p<0.005$ (FDR corrected), extant threshold $=10$ voxels.

Whole-brain regression was also performed across target groups on the simple contrast [Pain > No Pain] using on-line empathy ratings and IRI scores as behavioral covariates with a threshold of $p<0.05$, (FDR corrected), extant threshold $=10$ voxels.

Region of interest analyses were performed on a priori hypothesized regions-of-interest, specifically the dACC and bilateral AI, defined as a $6 \mathrm{~mm}$ sphere centered on peak voxels from a previous study on empathy for emotional pain in people $[\mathrm{d} \underline{\mathrm{ACC}}:-6,14,49 ; \underline{\mathrm{R}} \mathrm{AI}: 39,18,2 ; \underline{\mathrm{L}}$ AI: -48, 14, -8 (Mathur et al., 2010)] with the MarsBar toolbox in SPM2 (Brett, Anton, 
NEURAL RESPONSE TO PAIN OF PEOPLE, ANIMALS AND NATURE

Valabregue, \& Poline, 2002) (Figure 1). MNI coordinates were converted into Talairach space using a non-linear transformation (http://imaging.mrc-cbu.cam.ac.uk/imaging/MniTalairach). Brodmann areas and brain regions were identified based on the Talairach Atlas (Talairach \& Tournoux, 1988).

\section{Results}

\subsection{Behavioral results}

\section{$\underline{3.1 .1 \text { Analysis of variance }}$}

Two separate 2 (valence of experience: Pain, Neutral) x 3 (target group: People, Animals, Nature) repeated measures ANOVAs were conducted on empathy ratings and response reaction time during scanning.

\subsubsection{Empathy ratings}

There were significant main effects of both valence of experience $[F(1,14)=118.42, p<$ $0.001]$, and target group $[F(2,28)=9.35, p=0.001]$ on empathy ratings. There was also a significant interaction between valence and target group $[F(2,28)=8.33, p=0.001]$ such that all participants reported feeling more empathy toward pain relative to neutral scenes across target group [People: $t(14)=13.65, p<0.001, d=4.62$; Animals: $t(14)=9.52, p<0.001, d=2.92$;

Nature: $t(14)=7.66, p<0.001, d=2.77$ ], (Table 2), and people felt more relative empathy (Pain - Neutral) toward people $[t(14)=2.97, p=.01, d=0.60]$ and animals $[t(14)=4.98, p<0.001, d$ $=0.72]$ compared to nature. However, there was no significant difference in empathy ratings toward people compared to animals $[t(14)=-1.02, p=0.33, d=-0.11]$.

\subsubsection{Reaction time}


NEURAL RESPONSE TO PAIN OF PEOPLE, ANIMALS AND NATURE

There were also significant main effects of valence of experience on reaction time $[F(1$, 14) $=42.09, p<0.001]$, such that all participants were slower to rate empathy for pain relative to neutral scenes across target group [People: $t(14)=2.88, p=0.01, d=0.61$; Animals: $t(14)=$ $5.50, p<0.001, d=1.11$; Nature: $t(14)=8.72, p<0.001, d=1.15]$; and target group $[F(2,28)=$ 9.18, $p=0.001]$, such that participants were slower to rate empathy for scenarios depicting people relative to animals $[t(14)=3.46, p=0.004, d=0.42]$ and nature $[t(14)=4.01, p=0.001$, $d=0.58]$ (Table 2). There was also a significant interaction between valence and target group $[F(2,28)=3.64, p=0.04]$

\subsubsection{Correlations}

Relative empathy ratings (Pain - Neutral) were significantly correlated across domains $(\alpha$ $=0.88)$, such that participants who reported more empathy for people also did so for animals $(R$ $=0.58, p=0.02)$ and nature $(R=0.73, p=0.002)$. Similarly, when empathy for only pain scenarios was considered, this positive relationship between relative empathy for people and animals $(R=0.60, p=0.02)$ and people and nature $(R=0.73, p=0.002)$ remains. However, selfreported empathy ratings were not associated with any IRI subscales.

\subsection{Neuroimaging results}

\subsubsection{Main effect of pain}

As expected, the main effect of pain recruited brain areas associated with the processing of pain and empathy. Across participants and target groups, viewing and empathizing with painful scenarios was associated with activity in the dACC (extending to midcingulate cortex $\left(\right.$ aMCC) and supplementary motor area $\left.(\mathrm{SMA})^{3}\right)$, bilateral AI, and thalamus. Because of the robustness of this activity when collapsing across target groups, we report the results for the main effect of pain at the more stringent threshold of $p<0.05$ (FWE corrected), extant threshold 
NEURAL RESPONSE TO PAIN OF PEOPLE, ANIMALS AND NATURE

$=10$ voxels $($ Table 3$)$.

\subsubsection{People Pain > Neutral}

Consistent with prior research, the main effect of pain among human targets recruited brain areas associated with the processing of pain and empathy. Across all participants, viewing people in emotionally painful scenarios, relative to people in neutral scenarios, was associated with increased activation in bilateral AI, dACC, and thalamus (Table 4, Figure 1). These results are consistent with prior results of neural empathic response toward people in pain, and also provide independent replication of our prior results of neural reactivity in response to people in emotionally painful scenarios (Mathur et al., 2010).

\subsubsection{Animals Pain > Neutral}

Witnessing animals in physically painful, relative to neutral, scenes was also associated with increased reactivity within the bilateral AI, dACC, and thalamus (Table 5, Figure 1).

\subsubsection{Nature Pain > Neutral}

Witnessing harm being done to nature, relative to neutral nature scenes, was also associated with increased reactivity within the bilateral AI, dACC, and thalamus (Table 6, Figure 1).

\subsubsection{Main effect of target group}

A main effect of target group was only found when comparing animal scenes to nature scenes. Regions of the bilateral inferior temporal and occipital gyri, including the bilateral fusiform, were more active when viewing scenes depicting animals relative to scenes depicting nature (Table S1). No other main effects of target group were found.

\subsubsection{Interaction of pain and target group}

No suprathreshold interactions between pain and target group were found. 
NEURAL RESPONSE TO PAIN OF PEOPLE, ANIMALS AND NATURE

\subsubsection{Regression analysis}

Whole-brain regression analyses using empathy ratings and IRI scores as covariates did not reveal suprathreshold clusters.

\subsubsection{Region of interest analysis}

To test our hypotheses, we conducted a 2 (valence of experience: Pain, Neutral) x 3 (target group: People, Animals, Nature) repeated measures ANOVA was conducted on percent signal change within each of the a priori defined ROIs. As expected, there was a main effect of valence within each ROI, such that the dACC and bilateral AI were more reactive when empathizing with pain, relative to neutral, scenes across all target groups $[\mathrm{d} \underline{\mathrm{ACC}}: F(1,14)=$ $66.44, p<0.001 ; \underline{\mathrm{LAI}}: F(1,14)=30.77, p<0.001$; $\underline{\mathrm{R} \mathrm{AI}}: F(1,14)=29.29, p<0.001]$.

Interestingly, there was no effect of target group on activity within regions of interest (Figure 1). However, within the R AI, there was a significant interaction between valence and target group $[F(2,28)=3.74, p=0.04]$ such that the mean difference between pain and neutral $\mathrm{R}$ AI activity was smaller in response to human, relative to animal scenes $[t(15)=2.69, p=0.02, d$ $=0.72]$. This difference was driven by significantly greater R AI reactivity in response to neutral people, relative to neutral animal and nature, scenes $[F(2,28)=3.46, p=0.05]$. There were no significant differences within the pain condition (all $p \mathrm{~s}>0.05$ ).

Activity within the ROIs in response to pain, relative to neutral, scenes was not significantly correlated with online empathy ratings or IRI total and subscale scores (all $p \mathrm{~s}>$ 0.05). However, dACC and bilateral AI activity was significantly and positively correlated within each target domain (Table S2).

\section{Discussion}




\section{NEURAL RESPONSE TO PAIN OF PEOPLE, ANIMALS AND NATURE}

The present results demonstrate overlapping neural representation underlying pain perceived in human and non-human entities, specifically animals and nature. Our behavioral findings demonstrate that people report a negative affective response to not only the pain of other people, but also pain or harm of animals and nature. Importantly, neural response within brain areas (e.g. dACC, bilateral AI) consistently associated with pain perception and empathy for the pain of other people was similar when responding to the pain or suffering perceived in animals or the natural environment. This extends prior research that demonstrated overlapping dACC and bilateral AI activity in response to the pain of other people and the non-human species arguably most often empathized with by humans - dogs (Franklin et al., 2013). Hence, the current evidence lends support to our first hypothesis that the perception of pain and suffering relies upon a shared neural network for human and non-human entities. Convergent support of this hypothesis comes from the strong association between participants' subjective affective response toward humans, animals and nature in our study, suggesting a common psychological process underlying the perception of and response to the pain or harm of all three targets.

One reason response to the pain or harm of non-human entities in the natural world may rely on processes associated with human pain perception and response is that both of these contexts signal a need for action, whether it be to rescue others or retreat from danger. Empathy for people has long been understood as a key motivator of helping behavior (Batson et al., 1991; Preston \& de Waal, 2002). The perception-action model of empathy highlights the automaticity of this process and its relevance for survival of the species (Preston \& de Waal, 2002). Similarly, perception and response to the pain or harm of animals and nature may also have had survival advantages for humans. From the first year of life, people are exposed to cultural models of nature emphasizing an interdependent relation between humans and the surrounding 
NEURAL RESPONSE TO PAIN OF PEOPLE, ANIMALS AND NATURE

biological world (Bang, Medin, Atran, 2007; Hermann, Waxman, Medin, 2010). The cultivation and harvesting of, and general interdependence between humans, animals and the natural environment for survival also necessitated preserving these entities, which may have required the ability to identify harm or damage being caused to these targets and motivation to respond to alleviate such harm and damage.

Another reason that pain perception for non-humans may rely upon a shared neural network may be due to an automatic process whereby people rely upon person perception processes and human-related empathic processing in order to empathize with non-humans (Epley, Waytz, and Cacioppo, 2007). If this is an automatic or default processing strategy, then it may also result in preservation motives. Others have suggested that the process of anthropomorphizing non-human entities may lead to treating those entities as moral agents worthy of respect and concern (Epley, Waytz, and Cacioppo, 2007). Future studies should be conducted to tease apart whether the psychological process of anthropomorphizing non-human targets in pain or being harmed recruits the $\mathrm{dACC}$ and bilateral AI, or if this activity is relatively automatically recruited in response to such targets. While it is possible that anthropomorphism is necessary for this pattern of activity in response to the pain or harm of non-humans, it is also possible that this common activity is what allows for the affective response to the pain or harm of various human and non-human targets, but that activity in other neural regions, such as medial prefrontal cortex or temporoparietal junction, may allow for the more cognitive process of attributing agency to non-human targets. Discovery of the independent predictive value of, as well as interactions between, these processes on helping behavior is also needed. Future studies that additionally examine brain responses related to "positive empathy" (Morelli, Lieberman, and Zaki, 2015) for humans and non-human species may shed light on these competing hypotheses 
NEURAL RESPONSE TO PAIN OF PEOPLE, ANIMALS AND NATURE

by determining the role of context (positive vs. negative) and target domain (human, animal, nature) on the degree of overlapping activations. For instance, if anthropomorphic processes underlie empathy for non-human entities, we would expect overlap in positive empathic response across target domains.

Limitations of the present study include the lack of correlation between empathy ratings and $\mathrm{dACC}$ and AI activity, possibly because this activity may correspond to a more general psychological process such as the salience of the harmful scenes. Therefore, we more conservatively interpret this activity as neural response to the pain or harm of each target. Importantly, this neural response is routinely interpreted as the brain response to empathy. Future studies should aim to further explore the contribution of the ACC and AI to empathic processing, and whether, and in what ways, this activity differs from activity associated with perceiving and responding to harm or aversive scenes more generally. In response to reverse inferences being made about the function of the AACC and AI, others have argued that activation in these regions can be fully explained by stimuli saliency (Iannetti et al., 2013). It is possible that our results reflect a salience effect across domains. However, given the fundamental salience of human stimuli (Van Overwalle, 2009), if our observed results were due solely to salience effects, we would expect greater response to the human, relative to animal and nature, scenes. Nonetheless, follow up studies should control for the effects of salience differences across domains. Future studies should also extend this exploration to examine the neural and behavioral response to the destruction or damage of non-natural items which may also be salient and communicate threat, but may be less likely to evoke empathic response. It is also possible that assessing empathy toward non-human targets triggers empathic processes. However, prior research suggests that similar patterns of neural empathic responses are evoked when empathy is 
NEURAL RESPONSE TO PAIN OF PEOPLE, ANIMALS AND NATURE

explicitly assessed through similar instructions (e.g., Cheon et al., 2011; Mathur et al., 2010), as well as when attention to the pain of human and animal targets is implicit or incidental (Cheon et al., 2013; Franklin et al., 2013). Therefore, it is unlikely that our results are contingent on task instruction. Finally, an important consideration is that our small sample size limits detection of significant differences. We chose a detection threshold optimized to avoid false-positive results, but cannot completely rule out the possibility of false-negative results. As such, we caution interpretation of non-significant main effects. Here we focus on the robust and novel finding that neural response within brain areas that have become known for their association with (and whose activation is often interpreted as) pain perception and empathy are recruited not only in response to the pain of other humans, but also to the perceived pain or harm of animals or the natural environment. The present study provides a foundation for future investigations into the nuanced differences in the neural response to these different target groups.

This study offers new insights about the dACC and AI activity in response to the pain of other people. Numerous prior studies have demonstrated activity within these regions to be associated with empathy toward the physical and emotional pain of other people, yet the specificity of this neural signature in the context of response to the pain of others is less well understood. Recent critiques have cautioned against assumptions and interpretations regarding the specificity of this activity within the dACC and AI (Decety, 2010; Schleim \& Roiser, 2009). Research is needed to test the nature of this activity when responding to human and non-human targets in various situations, and whether involvement of this activity is associated with subjective empathic feelings, intensity of observed pain or harm, saliency of the harmful pictures, or some other factor. The present is a first study testing the neural response to the pain or harm of not just humans, but also animals and nature. The result of similar activity across target types 
NEURAL RESPONSE TO PAIN OF PEOPLE, ANIMALS AND NATURE

suggests that this activity is not as specific as the literature up to this point has assumed. We encourage readers to interpret the present results from this novel study as a first step. The finding that $\mathrm{dACC}$ and $\mathrm{AI}$ activity is similar when perceiving and responding to pain or harm of human, animal, and nature targets does not preclude the possibility that there are differences that were not detected with the current paradigm. However, we believe the present results are particularly important given the novelty of the inclusion of various biological targets, and the recent surge of studies on the neuroscience of empathy. It is possible that 1) the neural empathic process is not specific to humans, and future studies should determine the bounds of this extension by using targets that vary on dimensions of similarity, mental capacity, value to humans, renewability and other hypothesized modulators; or 2) that the AACC and AI component of empathy is more promiscuous that previously thought and rather represents a more general process underlying empathy and the perception of harm. Rather than assuming specificity, future studies should test the component contribution of this network to empathy.

One important consideration for future studies is to further explore the functional specificity of empathic neural circuitry. Prior studies have demonstrated the flexibility of this system in detecting salience in the external environment. The present study demonstrates that empathy for human and non-human entities such as animals and nature evokes a similar brain response. Hence, our findings suggest a general, rather than specific or human-centric, neural responding to perceived pain in one's social and natural environment. More broadly, our findings may provide a foundation for future studies examining the extent to which activity within these regions relies on anthropomorphic thinking, or is simply an automatic affective response to harmful situations, as well as the role that empathic neural circuitry plays in facilitating broad charitable action for living things in the natural world. 
NEURAL RESPONSE TO PAIN OF PEOPLE, ANIMALS AND NATURE

\section{Acknowledgements}

We thank A. Waytz, G. Bebko, L. Hechtman, and N. Pornpattananangkul for helpful feedback. 
NEURAL RESPONSE TO PAIN OF PEOPLE, ANIMALS AND NATURE

\section{Abbreviations}

dACC - dorsal anterior cingulate cortex

AI - anterior insula

ROI - region of interest 
NEURAL RESPONSE TO PAIN OF PEOPLE, ANIMALS AND NATURE

\section{Footnotes}

${ }^{1}$ To refer to the egregiously negative situations thought to evoke empathic response, we use the term pain when referring to humans and harm when referring to animals and nature.

${ }^{2}$ We refer to the stimuli depicting the pain or harm of people, animals, and nature, as Pain, and the control stimuli as Neutral.

${ }^{3}$ Though we refer to this large cluster as dACC throughout based on the location of peak voxels, this cluster is large across stimulus domains and, similar to meta-analytic descriptions of empathy-related brain response (Fan et al., 2011), extends to include the anterior MCC and SMA. 
NEURAL RESPONSE TO PAIN OF PEOPLE, ANIMALS AND NATURE

\section{References}

Amaro, E. \& Barker, G.J. (2006). Study design in fMRI: Basic principles. Brain and Cognition, 60: $220-232$.

Bang, M., Medin, D. L., \& Atran, S. (2007). Cultural mosaics and mental models of nature. Proceedings of the National Academy of Sciences, 104(35), 13868.

Batson, C. D., Batson, J. G., Slingsby, J. K., Harrell, K. L., Peekna, H. M., \& Todd, R. M. (1991). Empathic joy and the empathy-altruism hypothesis. Journal of Personality and Social Psychology, 61(3), 413-426.

Birn, R.M., Cox, R.W., \& Bandettini, P.A. (2002). Detection versus Estimation in EventRelated fMRI: Choosing the Optimal Stimulus Timing. Neuroimage, 15, 252-264.

Botvinick, M., Jha, A. P., Bylsma, L. M., Fabian, S. A., Solomon, P. E., \& Prkachin, K. M. (2005). Viewing facial expressions of pain engages cortical areas involved in the direct experience of pain. Neuroimage, 25(1), 312-319.

Brett, M., Anton, J. L., Valabregue, R., \& Poline, J.-B. (2002). Region of interest analysis using an SPM toolbox. Neuroimage, 16, abstract 497.

Charities Aid Foundation (2011). The world giving index. Retrieved from http://www.cafonline.org.

Cheon, B. K., Im, D., Harada, T., Kim, J. S., Mathur, V. A., Scimeca, J. M., et al. (2011). Cultural influences on neural basis of intergroup empathy. Neuroimage, 57(2), 642-650.

Cheon, B.K., Im, D., Harada, T., Kim, J., Mathur, V.A., Scimeca, J., et al. (2013). The cultural modulation of the neural correlates of empathy: The role of 'other-focusedness.' Neuropsychologia, 51(7), 1177-1186. 
NEURAL RESPONSE TO PAIN OF PEOPLE, ANIMALS AND NATURE

Chiao, J. Y., Mathur, V. A., Harada, T., \& Lipke, T. (2009). Neural basis of preference for human social hierarchy versus egalitarianism. Annals of the New York Academy of Sciences, 1167, 174-181.

Davis, M. (1980). Interpersonal reactivity index. Washington, DC: American Psychological Association.

Decety, J. (2010). To what extent is the experience of empathy mediated by shared neural circuits? Emotion Review, 2(3), 204-207.

Decety, J., Echols, S., \& Correll, J. (2010). The blame game: the effect of responsibility and social stigma on empathy for pain. Journal of Cognitive Neuroscience, 22(5), 985-997.

Epley, N., Waytz, A., \& Cacioppo, J. T. (2007). On seeing human: A three-factor theory of anthropomorphism. Psychological Review, 114(4), 864.

Fan, Y., Duncan, N. W., de Greck, M., \& Northoff, G. (2011). Is there a core neural network in empathy? An fMRI based quantitative meta-analysis. Neuroscience \& Biobehavioral Reviews, 35(3), 903-911.

Franklin, R.G., Nelson, A.J., Baker, M., Beeney, J.E., Vescio, T.K., Lenz-Watson, A., \& Adams Jr., R.B. (2013). Neural responses to perceiving suffering in humans and animals. Social Neuroscience, 8(3), 217-27.

Friston, K. J., Holmes, A. P., Worsley, K. J., Poline, J. P., Frith, C. D., \& Frackowiak, R. S. J. (1994). Statistical parametric maps in functional imaging: A general linear approach. Human Brain Mapping, 2(4), 189-210.

Herrmann, P., Waxman, S. R., \& Medin, D. L. (2010). Anthropocentrism is not the first step in children's reasoning about the natural world. Proceedings of the National Academy of Sciences, 107(22), 9979-9984. 
NEURAL RESPONSE TO PAIN OF PEOPLE, ANIMALS AND NATURE

Hills, A. M. (1995). Empathy and belief in the mental experience of animals. Anthrozoos: A Multidisciplinary Journal of The Interactions of People \& Animals, 8(3), 132-142.

Iannetti, G. D., Salomons, T. V., Moayedi, M., Mouraux, A., \& Davis, K. D. (2013). Beyond metaphor: contrasting mechanisms of social and physical pain.Trends in cognitive sciences, 17(8), 371-378.

Jackson, P. L., Rainville, P., \& Decety, J. (2006). To what extent do we share the pain of others? Insight from the neural bases of pain empathy. Pain, 125(1-2), 5-9.

Lamm, C., Decety, J., \& Singer, T. (2011). Meta-analytic evidence for common and distinct neural networks associated with directly experienced pain and empathy for pain. Neuroimage, 54, 2492-2502.

Lamm, C., Nusbaum, H. C., Meltzoff, A. N., \& Decety, J. (2007). What are you feeling? Using functional magnetic resonance imaging to assess the modulation of sensory and affective responses during empathy for pain. PLoS ONE, 2(12), e1292.

Masten, C. L., Morelli, S. A., \& Eisenberger, N. I. (2011). An fMRI investigation of empathy for 'social pain' and subsequent prosocial behavior. Neuroimage, 55(1), 381-388

Mathur, V. A., Harada, T., \& Chiao, J. Y. (2012). Racial identification modulates default network activity for same and other races. Human Brain Mapping, 33(8), 18831893.Mathur, V. A., Harada, T., Lipke, T., \& Chiao, J. Y. (2010). Neural basis of extraordinary empathy and altruistic motivation. Neuroimage, 51(4), 1468-1475.

Mitchell, J. P., Macrae, C. N., \& Banaji, M. R. (2005). Forming impressions of people versus inanimate objects: Social-cognitive processing in the medial prefrontal cortex. Neuroimage, 26, 251-257.

Morelli, S. A., Lieberman, M. D., \& Zaki, J. (2015). The emerging study of positive empathy. Social and Personality Psychology Compass, 9(2), 57-68. 
NEURAL RESPONSE TO PAIN OF PEOPLE, ANIMALS AND NATURE

Phillips, C. (2009). Empathy Towards Animals. The Welfare of Animals, 47-54.

Preston, S. D., \& de Waal, F. B. M. (2002). Empathy: Its ultimate and proximate bases. Behav Brain Sci, 25(1), 1-20.

Rae Westbury, H., \& Neumann, D. L. (2008). Empathy-related responses to moving film stimuli depicting human and non-human animal targets in negative circumstances. Biological Psychology, 78(1), 66-74.

Saarela, M. V., Hlushchuk, Y., Williams, A. C. d. C., Schurmann, M., Kalso, E., \& Hari, R. (2007). The Compassionate Brain: Humans Detect Intensity of Pain from Another's Face. Cereb. Cortex, 17(1), 230-237.

Saxe, R., \& Kanwisher, N. (2003). People thinking about people: The role of the temporoparietal junction in "theory of mind." Neuroimage, 19, 1835-1842.

Schleim, S. \& Roiser, J.P. (2009). fMRI in translation: The challenges facing real-world applications. Frontiers in Human Neuroscience, 3,63.

Schultz, P. W. (2000). Empathizing with nature: The effects of perspective taking on concern for environmental issues. Journal of Social Issues, Promoting Environmentalism, 56, 391.

Schultz, P. W. (2001). The structure of environmental concern: Concern for self, other people, and the biosphere. Journal of Environmental Psychology, 21(4), 327-339.

Singer, T., Seymour, B., O'Doherty, J., Kaube, H., Dolan, R. J., \& Frith, C. D. (2004). Empathy for pain involves the affective but not sensory components of pain. Science, 303(5661), $1157-1162$

Singer, T., Seymour, B., O'Doherty, J. P., Stephan, K. E., Dolan, R. J., \& Frith, C. D. (2006). Empathic neural responses are modulated by the perceived fairness of others. Nature, 439(7075), 466-469. 


\section{NEURAL RESPONSE TO PAIN OF PEOPLE, ANIMALS AND NATURE}

Talairach, J., \& Tournoux, P. (1988). Co-planar stereotaxic atlas of the human brain : 3dimensional proportional system : an approach to cerebral imaging. New York, NY: Thieme Medical Publishers.

Texas Department of Transportation. (2001). "If your mother were Texas". Don't Mess with Texas. Austin, TX. Available at http://dontmesswithtexas.org/view-ads/view-all-ads/.

The Humane Society of the United States. (2011). Rescue Animals Now. Washington, DC. Available at https://www.humanesociety.org/

Van Overwalle, F. (2009). Social cognition and the brain: A meta-analysis. Human Brain Mapping, 30, 829-858.

Wagner, D. D., Kelley, W. M., \& Heatherton, T. F. (2011). Individual differences in the spontaneous recruitment of brain regions supporting mental state understanding when viewing natural social scenes. Cerebral Cortex, 21(12), 2788-2796.

Xu, X., Zuo, X., Wang, X., \& Han, S. (2009). Do you feel my pain? Racial group membership modulates empathic neural responses. Journal of Neuroscience, 29(26), 8525-8529.

Zaki, J., Ochsner, K. N., Hanelin, J., Wager, T. D., \& Mackey, S. C. (2007). Different circuits for different pain: Patterns of functional connectivity reveal distinct networks for processing pain in self and others. Social Neuroscience, 2(3), 276 - 291. 


\section{People}
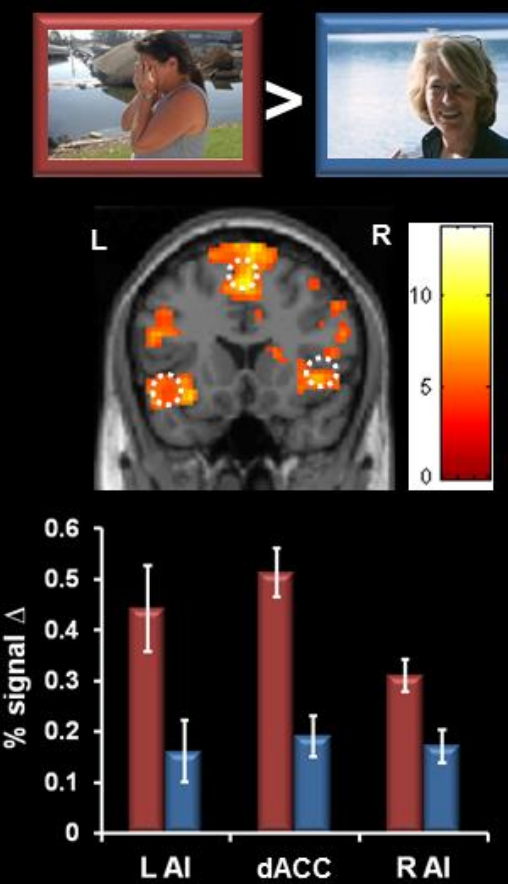

Animals

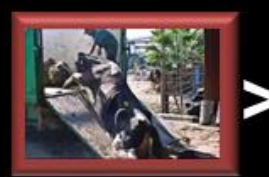

L
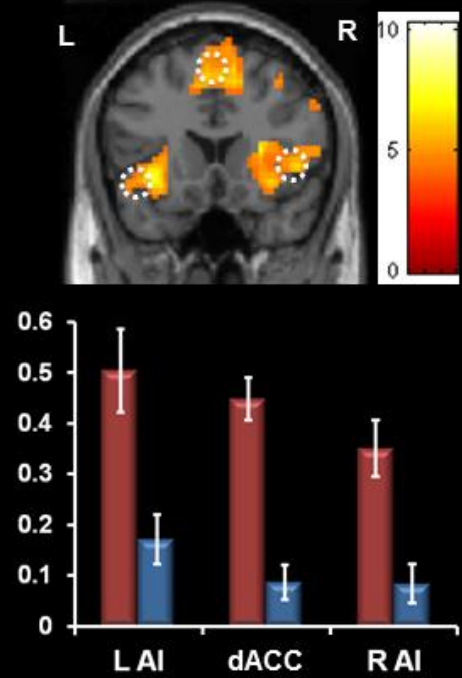

× Pain $\leq$ Neutral

\section{Nature}
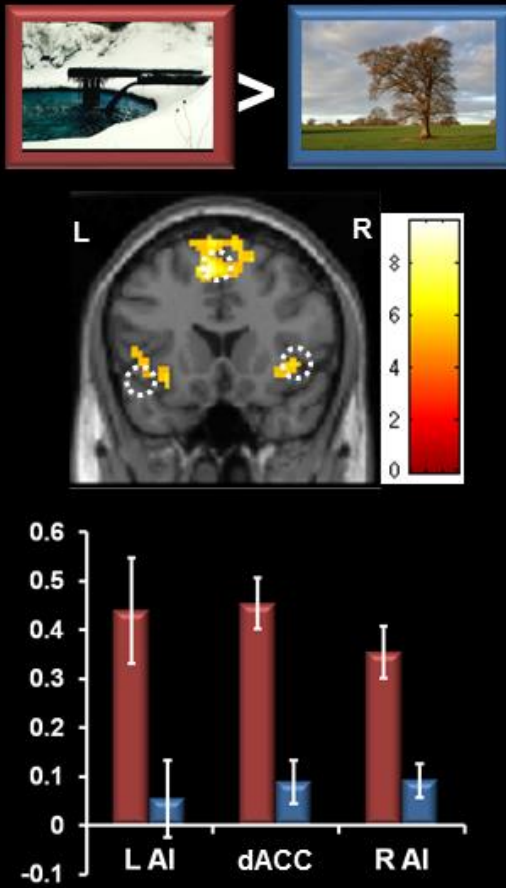

Figure 1: Main effect of pain (Pain $>$ Neutral), $\mathrm{y}=20, p<0.005$ (FDR corrected), $k=10$.

Dotted circles outline a priori ROIs from which percent signal change was extracted. 
NEURAL RESPONSE TO PAIN OF PEOPLE, ANIMALS AND NATURE

Table 1: Behavioral validation experiment results

\begin{tabular}{rcc|cc|cc}
\multicolumn{2}{c}{ People } & \multicolumn{2}{c}{ Animals } & \multicolumn{2}{c}{ Nature } \\
\hline \multirow{2}{*}{ Pain } & $5.00 \pm 0.19^{*}$ & $0.45 \pm 0.11$ & $5.42 \pm 0.29^{*}$ & $0.56 \pm 0.11$ & $4.76 \pm 0.26^{*}$ & $\underline{\text { Pain }}$ \\
\cline { 3 - 4 } Agency & $2.66 \pm 0.31^{*}$ & $4.64 \pm 0.39$ & $0.55 \pm 0.22^{*}$ & $1.38 \pm 0.31$ & $0.88 \pm 0.25$ & $0.82 \pm 0.26$ \\
Empathy & $4.81 \pm 0.22^{*}$ & $0.33 \pm 0.10$ & $6.03 \pm 0.13^{*}$ & $1.02 \pm 0.20$ & $4.49 \pm 0.29 *$ & $0.42 \pm 0.11$ \\
\hline
\end{tabular}

Note: Ratings from $0-7$; Mean \pm SE; *, $\mathrm{p}<0.05$ when compared with the Neutral condition

Table 2: Behavioral results

\begin{tabular}{ccc|cc|cc}
\hline \multicolumn{2}{c}{ People } & \multicolumn{2}{c}{ Animals } & \multicolumn{2}{c}{ Nature } \\
\hline \multirow{2}{*}{ Empathy } & $\underline{\text { Pain }}$ & $\underline{\text { Neutral }}$ & $\underline{\text { Pain }}$ & $\underline{\text { Neutral }}$ & $\underline{\text { Pain }}$ & $\underline{\text { Neutral }}$ \\
RT (ms) & $914.26 \pm 63.85^{*}$ & $774.61 \pm 54.21$ & $888.36 \pm 74.16^{*}$ & $621.06 \pm 47.42$ & $837.95 \pm 48.11^{*}$ & $623.95 \pm 48.27$ \\
\hline
\end{tabular}

Note: Ratings from 1-4; Mean $\pm \mathrm{SE}$; ${ }^{*}, p<0.05$ when compared with the Neutral condition 


\begin{tabular}{ccccccl}
\hline \multicolumn{1}{c}{ Table 3. Main effect of pain: All target groups (Pain $>$ Neutral) } \\
\hline$X$ & $Y$ & $Z$ & Z score & Voxels & BA & Brain area \\
3 & 29 & 54 & 5.82 & 143 & $6,8,32$ & dACC \\
0 & -24 & -11 & 5.7 & 187 & & Midbrain \\
33 & -60 & -25 & 5.7 & 48 & & R Cerebellum \\
-30 & 20 & -9 & 5.48 & 43 & & L AI \\
30 & -50 & -5 & 5.44 & 29 & 19 & R Parahippocampal Gyrus \\
36 & 23 & -9 & 5.38 & 22 & & R AI \\
12 & -6 & 6 & 5.14 & 17 & & Thalamus \\
\hline \multicolumn{7}{c}{}
\end{tabular}

$p<0.05$ (FWE corrected), $k>10$

\begin{tabular}{ccccccl}
\hline \multicolumn{6}{c}{ Table 4 . Main effect of pain: People (Pain > Neutral) } \\
\hline$X$ & $Y$ & $Z$ & Z score & Voxels & BA & Brain area \\
$\mathbf{- 3 0}$ & $\mathbf{2 3}$ & $\mathbf{- 6}$ & $\mathbf{6 . 0 3}$ & $\mathbf{2 6 8}$ & $\mathbf{1 4 7}$ & L AI/Inferior Frontal Gyrus \\
$\mathbf{3}$ & $\mathbf{1 4}$ & $\mathbf{5 5}$ & $\mathbf{5 . 4 6}$ & $\mathbf{1 0 4 5}$ & $\mathbf{3 2 / 6}$ & dACC/Superior Frontal Gyrus \\
12 & -61 & 53 & 5.27 & 573 & 7 & R Superior Parietal Lobule \\
$\mathbf{5 6}$ & $\mathbf{1 3}$ & $\mathbf{2 1}$ & $\mathbf{5 . 1 7}$ & $\mathbf{4 5 0}$ & $\mathbf{1 4 5}$ & R AI/Inferior Frontal Gyrus \\
42 & 33 & 23 & 5.11 & 119 & 46 & R Middle Frontal Gyrus \\
-21 & -73 & -9 & 5.11 & 1067 & 18 & L Lingual Gyrus \\
-42 & -84 & 10 & 5.07 & 171 & 19 & L Middle Occipital Gyrus \\
-12 & 0 & 8 & 5.03 & 617 & & L Thalamus/Putamen \\
-33 & 5 & 36 & 4.96 & 307 & 9 & L Precentral Gyrus \\
-27 & -71 & 45 & 4.96 & 134 & 7 & L Superior Parietal Lobule \\
50 & -26 & -4 & 4.86 & 58 & 21 & R Superior Temporal Gyrus \\
-30 & 3 & 52 & 4.75 & 108 & 6 & L Middle Frontal Gyrus \\
-9 & -32 & -3 & 4.74 & 71 & & L Cerebellum \\
12 & -30 & -4 & 4.66 & 50 & & R Thalamus \\
-36 & 56 & 8 & 4.46 & 73 & 10 & L Middle Frontal Gyrus \\
-27 & -50 & -3 & 4.34 & 47 & 19 & L Parahippocampal Gyrus \\
-45 & 46 & -7 & 4.24 & 12 & 47 & L Middle Frontal Gyrus \\
21 & 59 & 22 & 4.23 & 37 & 10 & R Superior Temporal Gyrus \\
-36 & -54 & -28 & 4.06 & 27 & & L Cerebellum \\
-3 & 57 & 39 & 3.91 & 22 & 9 & L Middle Frontal Gyrus \\
-39 & -42 & 41 & 3.54 & 15 & 40 & L Inferior Parietal Lobule \\
\hline & & & & & &
\end{tabular}

$p<0.005$ (FDR corrected), $k>10$ 


\begin{tabular}{ccccccl}
\hline \multicolumn{6}{c}{ Table 5. Main effect of pain: Animals (Pain $>$ Neutral) } \\
\hline$X$ & $Y$ & $Z$ & Z score & Voxels & BA & Brain area \\
$\mathbf{- 6}$ & $\mathbf{- 1 8}$ & $\mathbf{- 9}$ & $\mathbf{5 . 4}$ & $\mathbf{4 4 0 0}$ & & Bilateral thalamus/R AI \\
$\mathbf{- 1 2}$ & $\mathbf{3 6}$ & $\mathbf{2 0}$ & $\mathbf{5 . 3}$ & $\mathbf{6 8 4}$ & $\mathbf{3 2}$ & dACC \\
27 & -75 & 20 & 4.94 & 368 & 31 & R Precuneus \\
$\mathbf{- 3 3}$ & $\mathbf{2 3}$ & $\mathbf{- 9}$ & $\mathbf{4 . 9 3}$ & $\mathbf{2 4 1}$ & & L AI \\
-42 & 2 & 30 & 4.79 & 58 & 9 & L Inferior Frontal Gyrus \\
-30 & -1 & 47 & 4.26 & 51 & 6 & L Middle Frontal Gyrus \\
39 & 54 & 22 & 4.19 & 44 & 10 & R Superior Frontal Gyrus \\
36 & 5 & 49 & 4.08 & 223 & 6 & R Middle Frontal Gyrus \\
0 & -31 & -36 & 3.93 & 22 & & Brainstem \\
-33 & 51 & 25 & 3.79 & 14 & 9 & L Superior Frontal Gyrus \\
\hline
\end{tabular}

$p<0.005$ (FDR corrected), $k>10$

\begin{tabular}{ccccccl}
\hline \multicolumn{6}{c}{ Table 6. Main effect of pain: Nature (Pain > Neutral) } \\
\hline$X$ & $Y$ & $Z$ & Z score & Voxels & BA & Brain area \\
0 & -29 & 1 & 5.25 & 198 & & Thalamus, Pulvinar \\
$\mathbf{- 6}$ & $\mathbf{2 0}$ & $\mathbf{4 6}$ & $\mathbf{5 . 1 2}$ & $\mathbf{3 9 6}$ & $\mathbf{3 2 / 8}$ & dACC/Medial Frontal Gyrus \\
36 & -60 & -25 & 4.61 & 77 & & R Cerebellum \\
-39 & -66 & -30 & 4.54 & 93 & & L Cerebellum \\
$\mathbf{5 0}$ & $\mathbf{1 5}$ & $\mathbf{1 0}$ & $\mathbf{4 . 5 2}$ & $\mathbf{1 0}$ & $\mathbf{1 4 4}$ & $\mathbf{R}$ AI/ Inferior Frontal Gyru \\
$\mathbf{- 4 8}$ & $\mathbf{1 5}$ & $\mathbf{5}$ & $\mathbf{4 . 4 1}$ & $\mathbf{6 4}$ & $\mathbf{1 4 4}$ & L AI/Inferior Frontal Gyrus \\
18 & 3 & 0 & 4.32 & 24 & & L Thalamus/Putamen \\
0 & -57 & -35 & 4.21 & 14 & & L Cerebellum \\
6 & -28 & 29 & 4.16 & 21 & 23 & R PCC \\
$\mathbf{4 2}$ & $\mathbf{1 7}$ & $\mathbf{- 3}$ & $\mathbf{4 . 1 5}$ & $\mathbf{4 2}$ & & R AI \\
36 & -50 & 47 & 4.15 & 13 & 40 & R Inferior Parietal Lobule \\
\hline
\end{tabular}

$p<0.005$ (FDR corrected), $k>10$ 
NEURAL RESPONSE TO PAIN OF PEOPLE, ANIMALS AND NATURE

\begin{tabular}{ccccccl}
\hline \multicolumn{6}{c}{ Table } & S1. Main effect of Agency: Animals \\
\hline$X$ & $Y$ & $Z$ & Z sain + Neutral) $>$ Nature & (Pain + Neutral) \\
45 & -70 & -2 & 5.68 & 276 & 19 & R Inferior Temporal Gyrus \\
-42 & -82 & -9 & 5.49 & 180 & 18 & L Inferior Occipital Gyrus \\
-42 & -60 & -17 & 4.35 & 24 & 37 & L Fusiform Gyrus/Middle Occipital Gyrus \\
45 & -59 & -17 & 4.21 & 20 & 37 & R Fusiform Gyrus/Middle Occipital Gyrus \\
\hline
\end{tabular}

$p<0.005$ (FDR corrected), $k>10$

Table S2: Neural Empathy Matrix Intercorrelation

\begin{tabular}{|c|c|c|c|c|c|c|}
\hline & \multicolumn{2}{|c|}{ People } & \multicolumn{2}{|c|}{ Animals } & \multicolumn{2}{|c|}{ Nature } \\
\hline & $\mathrm{dACC}$ & $\underline{\mathrm{LAI}}$ & dACC & $\underline{\mathrm{LAI}}$ & dACC & $\underline{\mathrm{LAI}}$ \\
\hline dACC & - & - & - & - & - & - \\
\hline$\underline{\mathrm{L} A \mathrm{I}}$ & $.70 * *$ & - & $.42^{\dagger}$ & - & $.59 *$ & - \\
\hline$\underline{\mathrm{R} \mathrm{AI}}$ & $.55^{*}$ & $.47^{\dagger}$ & $.82 * *$ & $.58^{*}$ & $.83 * *$ & $.69 * *$ \\
\hline
\end{tabular}

